MATHEMATICS OF COMPUTATION

Volume 77, Number 262, April 2008, Pages 757-771

S 0025-5718(07)02039-X

Article electronically published on November 13, 2007

\title{
SUPERCONVERGENCE ANALYSIS FOR MAXWELL'S EQUATIONS IN DISPERSIVE MEDIA
}

\author{
QUN LIN AND JICHUN LI
}

\begin{abstract}
In this paper, we consider the time dependent Maxwell's equations in dispersive media on a bounded three-dimensional domain. Global superconvergence is obtained for semi-discrete mixed finite element methods for three most popular dispersive media models: the isotropic cold plasma, the one-pole Debye medium, and the two-pole Lorentz medium. Global superconvergence for a standard finite element method is also presented. To our best knowledge, this is the first superconvergence analysis obtained for Maxwell's equations when dispersive media are involved.
\end{abstract}

\section{INTRODUCTION}

Recently there is a growing interest in finite element modeling and analysis of Maxwell's equations (e.g. [7, 5, 9, 13, 12, 15, 16, 33, 34, 8]). The readers can find more references in some recent books [2, 18, 35] and conference proceedings [1, 3, 10. However, most work is restricted to simple media such as free space. Very few papers are devoted to dispersive media using the finite element method (FEM), though there are some publications in finite-difference time-domain (FDTD) modeling of dispersive media started since 1990 [38, Ch. 9]. We want to remark that dispersive media are ubiquitous, for example human tissue, soil, snow, ice, plasma, fiber optics and radar-absorbing materials. In order to accurately perform wideband electromagnetic simulations, we have to consider the effect of medium dispersion in the modeling equations. Applications of time-domain finite element method (TDFEM) for dispersive media have appeared only very recently [17, 32]. However, there exists no theoretical error analysis except our initial efforts $[20,21,22$.

Superconvergence of FEM is a phenomenon that the convergence rate exceeds what general cases can provide. Since the 1970s, many studies have been conducted for superconvergence, which can be achieved for smoother solutions with structured grids. More details can be found in [27, 4, 30, 19, 39] and the references therein. In 1994, Monk 34 initiated the investigation on superconvergence for Maxwell's equations in simple media. Recently, Lin and his collaborators [29, 26] systematically developed global superconvergence, but still restricted it to simple media. To our best knowledge, there exists no work in the literature which studies the superconvergence error analysis of TDFEM for Maxwell's equations in dispersive media. This paper intends to make an initial effort in this direction. Here we develop

Received by the editor May 25, 2006 and, in revised form, January 26, 2007.

2000 Mathematics Subject Classification. Primary 65N30, 35L15, 78Mxx.

Key words and phrases. Maxwell's equations, dispersive media, superconvergence analysis.

(C)2007 American Mathematical Society 
global superconvergence error analysis for semi-discrete standard and mixed finite element methods for Maxwell's equations when dispersive media are involved. The proof uses the powerful integral identity technique developed by Lin's group in the early 1990s [31, 24, 25] and applied later to many areas [23, 40, 14, 29, 30].

The rest of the paper is organized as follows. In Section 2, some notation and preliminary results are introduced. Then in Section 3, a semi-discrete mixed finite element scheme is developed for the isotropic cold plasma model. Superclose estimates are obtained on the cubic Nédélec curl conforming element. Similar results are derived for one-pole Debye medium and two-pole Lorentz medium. In Section 4, global superconvergence is proved for all three dispersive media by using the integral identity technique. In Section 5, we extend the global superconvergence analysis to a standard finite element method.

\section{NotATION AND PRELIMINARY RESULTS}

In this paper, $C$ (sometimes with a sub-index) denotes a generic constant, which is independent of the finite element mesh size $h$. We will introduce some notation to be used in this paper. We define

$$
\begin{aligned}
H(\operatorname{curl} ; \Omega) & =\left\{\boldsymbol{v} \in\left(L^{2}(\Omega)\right)^{3} ; \nabla \times \boldsymbol{v} \in\left(L^{2}(\Omega)\right)^{3}\right\} \\
H^{\alpha}(\operatorname{curl} ; \Omega) & =\left\{\boldsymbol{v} \in\left(H^{\alpha}(\Omega)\right)^{3} ; \nabla \times \boldsymbol{v} \in\left(H^{\alpha}(\Omega)\right)^{3}\right\}, \\
H_{0}(\operatorname{curl} ; \Omega) & =\{\boldsymbol{v} \in H(\operatorname{curl} ; \Omega) ; \mathbf{n} \times \boldsymbol{v}=0 \text { on } \partial \Omega\},
\end{aligned}
$$

where $\alpha \geq 0$ is a real number, and $\left(H^{\alpha}(\Omega)\right)^{3}$ is the standard Sobolev space equipped with the norm $\|\cdot\|_{\alpha}$ and semi-norm $|\cdot|_{\alpha}$ in a bounded polyhedral domain $\Omega$ of $\mathcal{R}^{3}$. Specifically $\|\cdot\|_{0}$ will mean the $\left(L^{2}(\Omega)\right)^{3}$-norm. Also $H(\operatorname{curl} ; \Omega)$ and $H^{\alpha}(\operatorname{curl} ; \Omega)$ are equipped with norms

$$
\begin{aligned}
\|\boldsymbol{v}\|_{0, \text { curl }} & =\left(\|\boldsymbol{v}\|_{0}^{2}+\|\operatorname{curl} \boldsymbol{v}\|_{0}^{2}\right)^{1 / 2} \\
\|\boldsymbol{v}\|_{\alpha, \text { curl }} & =\left(\|\boldsymbol{v}\|_{\alpha}^{2}+\|\operatorname{curl} \boldsymbol{v}\|_{\alpha}^{2}\right)^{1 / 2}
\end{aligned}
$$

We assume that the domain $\Omega$ is covered with a regular cubic mesh $T_{h}$ of maximum diameter $h$. Our mixed finite element spaces are [36]:

(1) $U_{h}=\left\{\phi \in\left(L^{2}(\Omega)\right)^{3} ;\left.\phi\right|_{e} \in Q_{k, k-1, k-1} \times Q_{k-1, k, k-1} \times Q_{k-1, k-1, k}, \forall e \in T_{h}\right\}$,

(2) $V_{h}=\left\{\boldsymbol{\psi} \in H(\operatorname{curl} ; \Omega) ;\left.\boldsymbol{\psi}\right|_{e} \in Q_{k-1, k, k} \times Q_{k, k-1, k} \times Q_{k, k, k-1}, \forall e \in T_{h}\right\}$,

where $Q_{i, j, k}$ denotes the space of polynomials whose degrees are less than or equal to $i, j, k$ for $x, y, z$, respectively. Note that we have [33, p. 114]:

$$
\nabla \times V_{h} \subset U_{h}
$$

Let $P_{h} \boldsymbol{E} \in U_{h}$ be the standard $\left(L^{2}(\Omega)\right)^{3}$ projection operator defined as

$$
\left(P_{h} \boldsymbol{E}-\boldsymbol{E}, \boldsymbol{\phi}\right)=0, \quad \forall \boldsymbol{\phi} \in U_{h}
$$


Furthermore, we define the interpolation operator $\boldsymbol{H}^{I} \in V_{h}$, which satisfies 29, p. 163]

$$
\begin{gathered}
\int_{l_{i}}\left(\boldsymbol{H}-\boldsymbol{H}^{I}\right) \cdot \tau q d l=0, \quad \forall q \in P_{k}\left(l_{i}\right), i=1, \cdots, 12, \\
\int_{\sigma_{i}}\left(\left(\boldsymbol{H}-\boldsymbol{H}^{I}\right) \times \mathbf{n}\right) \cdot \boldsymbol{q} d \sigma=0, \quad \forall \boldsymbol{q} \in Q_{k-2, k-1}\left(\sigma_{i}\right) \times Q_{k-1, k-2}\left(\sigma_{i}\right), i=1, \cdots, 6, \\
\int_{e}\left(\boldsymbol{H}-\boldsymbol{H}^{I}\right) \cdot \boldsymbol{q} d e=0, \quad \forall \boldsymbol{q} \in Q_{k-1, k-2, k-2} \times Q_{k-2, k-1, k-2} \times Q_{k-2, k-2, k-1},
\end{gathered}
$$

where $l_{i}, \sigma_{i}$ are edges and faces of the element $e, \tau$ is the unit tangent vector along edge $l_{i}$, and $\mathbf{n}$ is the normal vector on face $\sigma_{i}$.

In this paper, we need the following proven results:

Lemma 2.1 ([29, Lemma 3.1]).

$$
\int_{\Omega}\left(\nabla \times\left(\boldsymbol{H}-\boldsymbol{H}^{I}\right)\right) \cdot \boldsymbol{\phi} d \Omega=O\left(h^{k+1}\right)\|\boldsymbol{H}\|_{k+2}\|\boldsymbol{\phi}\|_{0} \quad \forall \boldsymbol{\phi} \in U_{h} .
$$

Lemma 2.2 ([29, Lemma 3.2]).

$$
\int_{\Omega}\left(\boldsymbol{H}-\boldsymbol{H}^{I}\right) \cdot \boldsymbol{\psi} d \Omega=O\left(h^{k+1}\right)\|\boldsymbol{H}\|_{k+1}\|\boldsymbol{\psi}\|_{0} \quad \forall \boldsymbol{\psi} \in V_{h} .
$$

Lemma 2.3 ([37, p. 13]). Let $f \in L^{1}(0, T)$ be a non-negative function, and let $g$ and $\varphi$ be continuous functions on $[0, T]$. Moreover, $g$ is non-decreasing. If $\varphi$ satisfies

Then

$$
\varphi(t) \leq g(t)+\int_{0}^{t} f(\tau) \varphi(\tau) d \tau \quad \forall t \in[0, T]
$$

$$
\varphi(t) \leq g(t) \exp \left(\int_{0}^{t} f(\tau) d \tau\right) \quad \forall t \in[0, T]
$$

\section{Superclose estimates}

In this section, we shall develop the superclose estimates for all three popular dispersive media models.

3.1. Isotropic cold plasma. The governing equations that describe electromagnetic wave propagation in isotropic non-magnetized cold electron plasma are [11, 6]

$$
\begin{aligned}
& \epsilon_{0} \frac{\partial \boldsymbol{E}}{\partial t}=\nabla \times \boldsymbol{H}-\boldsymbol{J}, \\
& \mu_{0} \frac{\partial \boldsymbol{H}}{\partial t}=-\nabla \times \boldsymbol{E}, \\
& \frac{\partial \boldsymbol{J}}{\partial t}+\nu \boldsymbol{J}=\epsilon_{0} \omega_{p}^{2} \boldsymbol{E},
\end{aligned}
$$

where $\boldsymbol{E}$ is the electric field, $\boldsymbol{H}$ is the magnetic field, $\epsilon_{0}$ is the permittivity of free space, $\mu_{0}$ is the permeability of free space, $\boldsymbol{J}$ is the polarization current density, $\omega_{p}$ is the plasma frequency, and $\nu$ is the electron-neutral collision frequency. Solving (7) with the assumption that the initial electron velocity is zero leads to [6, equation (8)]

$$
\boldsymbol{J}(\boldsymbol{x}, t ; \boldsymbol{E})=\epsilon_{0} \omega_{p}^{2} e^{-\nu t} \int_{0}^{t} e^{\nu s} \boldsymbol{E}(\boldsymbol{x}, s) d s=\epsilon_{0} \omega_{p}^{2} \int_{0}^{t} e^{-\nu(t-s)} \boldsymbol{E}(\boldsymbol{x}, s) d s,
$$


where $\boldsymbol{x} \in \Omega$. In this paper we let $\Omega$ be a bounded polyhedral domain in $\mathcal{R}^{3}$ with boundary $\partial \Omega$ and unit outward normal $\mathbf{n}$.

Substituting (8) into (5), we obtain the following system for $\boldsymbol{E}$ and $\boldsymbol{H}$ :

$$
\begin{aligned}
& \epsilon_{0} \boldsymbol{E}_{t}-\nabla \times \boldsymbol{H}+J(\boldsymbol{E})=0 \text { in } \Omega \times(0, T), \\
& \mu_{0} \boldsymbol{H}_{t}+\nabla \times \boldsymbol{E}=0 \text { in } \Omega \times(0, T),
\end{aligned}
$$

where for simplicity we denote $J(\boldsymbol{E})=\boldsymbol{J}(\boldsymbol{x}, t ; \boldsymbol{E})$.

To complete the problem, we assume that the boundary of $\Omega$ is a perfect conductor [35]:

$$
\mathbf{n} \times \boldsymbol{E}=0 \quad \text { on } \quad \partial \Omega \times(0, T),
$$

and the initial conditions are

$$
\boldsymbol{E}(\boldsymbol{x}, 0)=\boldsymbol{E}_{0}(\boldsymbol{x}) \text { and } \boldsymbol{H}(\boldsymbol{x}, 0)=\boldsymbol{H}_{0}(\boldsymbol{x}) \quad \text { for any } \boldsymbol{x} \in \Omega,
$$

where $\boldsymbol{E}_{0}$ and $\boldsymbol{H}_{0}$ are some given functions. Furthermore, $\boldsymbol{H}_{0}$ satisfies

$$
\nabla \cdot\left(\mu_{0} \boldsymbol{H}_{0}\right)=0 \quad \text { in } \Omega, \quad \boldsymbol{H}_{0} \cdot \mathbf{n}=0 \quad \text { on } \partial \Omega .
$$

Assuming the existence of smooth solutions to (9)-(13), we obtain the weak formulation: find the solution $(\boldsymbol{E}, \boldsymbol{H}) \in\left[C^{1}\left(0, T ;\left(L^{2}(\Omega)\right)^{3}\right) \cap C^{0}(0, T ; H(\operatorname{curl} ; \Omega))\right]^{2}$ of (91)-(13) such that

$$
\begin{array}{rlcl}
\epsilon_{0}\left(\boldsymbol{E}_{t}, \boldsymbol{\phi}\right)-(\nabla \times \boldsymbol{H}, \boldsymbol{\phi})+(J(\boldsymbol{E}), \boldsymbol{\phi}) & =0 & \forall \boldsymbol{\phi} \in\left(L^{2}(\Omega)\right)^{3}, \\
\mu_{0}\left(\boldsymbol{H}_{t}, \boldsymbol{\psi}\right)+(\boldsymbol{E}, \nabla \times \boldsymbol{\psi}) & = & 0 & \forall \boldsymbol{\psi} \in H(\mathrm{curl} ; \Omega)
\end{array}
$$

for $0<t \leq T$ with the initial conditions (12). Notice that the boundary condition (11) is used in deriving (15) since $(\nabla \times \boldsymbol{E}, \boldsymbol{\psi})=\langle\mathbf{n} \times \boldsymbol{E}, \boldsymbol{\psi}\rangle_{\partial \Omega}+(\boldsymbol{E}, \nabla \times \boldsymbol{\psi})$.

Now we can construct our semi-discrete mixed method for solving (14)-(15): find $\left(\boldsymbol{E}^{h}, \boldsymbol{H}^{h}\right) \in\left[C^{1}\left(0, T ; \mathbf{U}_{h}\right) \cap C^{1}\left(0, T ; \mathbf{V}_{h}\right)\right]^{2}$ such that

$$
\begin{aligned}
\epsilon_{0}\left(\boldsymbol{E}_{t}^{h}, \boldsymbol{\phi}_{h}\right)-\left(\nabla \times \boldsymbol{H}^{h}, \boldsymbol{\phi}_{h}\right)+\left(J\left(\boldsymbol{E}^{h}\right), \boldsymbol{\phi}_{h}\right) & =0 \quad \forall \boldsymbol{\phi}_{h} \in \mathbf{U}_{h}, \\
\mu_{0}\left(\boldsymbol{H}_{t}^{h}, \boldsymbol{\psi}_{h}\right)+\left(\boldsymbol{E}^{h}, \nabla \times \boldsymbol{\psi}_{h}\right) & =0 \quad \forall \boldsymbol{\psi}_{h} \in \mathbf{V}_{h}
\end{aligned}
$$

for $0<t \leq T$, subject to the initial conditions

$$
\boldsymbol{E}^{h}(\boldsymbol{x}, 0)=P_{h} \boldsymbol{E}_{0}(\boldsymbol{x}) \text { and } \boldsymbol{H}^{h}(\boldsymbol{x}, 0)=\boldsymbol{H}_{0}^{I}(\boldsymbol{x}),
$$

where $\boldsymbol{H}_{0}^{I} \in V_{h}$ is the interpolation of $\boldsymbol{H}_{0}$ defined in $\S 2$. Note that (16)-(18) is a system of linear ordinary differential equations, which guarantees the existence and uniqueness of solutions.

Theorem 3.1. Let $(\boldsymbol{E}(t), \boldsymbol{H}(t))$ and $\left(\boldsymbol{E}^{h}(t), \boldsymbol{H}^{h}(t)\right)$ be the solutions of (14) -(15) and (16)-(18) at time $t$, respectively. Then there is a constant $C=C\left(\epsilon_{0}, \mu_{0}, \omega_{p}, \nu\right)$, independent of the mesh size $h$, such that

$$
\begin{aligned}
\mu_{0}\left\|\left(\boldsymbol{H}^{I}-\boldsymbol{H}^{h}\right)(t)\right\|_{0}^{2}+\epsilon_{0}\left\|\left(P_{h} \boldsymbol{E}-\boldsymbol{E}^{h}\right)(t)\right\|_{0}^{2} \\
\quad \leq C h^{2(k+1)} \int_{0}^{t}\left[\|\boldsymbol{H}(t)\|_{k+2}^{2}+\left\|\boldsymbol{H}_{t}(t)\right\|_{k+1}^{2}\right] d t,
\end{aligned}
$$

where $k$ is the degree of edge elements in the space $\boldsymbol{V}_{h}$. 
Proof. Subtracting (16)-(17) from (14)-(15) with $\phi=\phi_{h}$ and $\boldsymbol{\psi}=\boldsymbol{\psi}_{h}$, respectively, we have the error equations:

$$
\begin{aligned}
& \epsilon_{0}\left(\left(\boldsymbol{E}-\boldsymbol{E}^{h}\right)_{t}, \boldsymbol{\phi}_{h}\right)-\left(\nabla \times\left(\boldsymbol{H}-\boldsymbol{H}^{h}\right), \boldsymbol{\phi}_{h}\right) \\
& \quad+\left(J\left(\boldsymbol{E}-\boldsymbol{E}^{h}\right), \boldsymbol{\phi}_{h}\right)=0 \quad \forall \boldsymbol{\phi}_{h} \in \mathbf{U}_{h}, \\
& \mu_{0}\left(\left(\boldsymbol{H}-\boldsymbol{H}^{h}\right)_{t}, \boldsymbol{\psi}_{h}\right)+\left(\boldsymbol{E}-\boldsymbol{E}^{h}, \nabla \times \boldsymbol{\psi}_{h}\right)=0 \quad \forall \boldsymbol{\psi}_{h} \in \mathbf{V}_{h} .
\end{aligned}
$$

Denote $\xi(t)=\left(P_{h} \boldsymbol{E}-\boldsymbol{E}^{h}\right)(t), \eta(t)=\left(\boldsymbol{H}^{I}-\boldsymbol{H}^{h}\right)(t)$. Choosing $\boldsymbol{\phi}_{h}=\xi, \boldsymbol{\psi}_{h}=\eta$ in (20)-(21), and rearranging terms lead to

$$
\begin{gathered}
\epsilon_{0}\left(\xi_{t}, \xi\right)-(\nabla \times \eta, \xi)=\epsilon_{0}\left(\left(P_{h} \boldsymbol{E}-\boldsymbol{E}\right)_{t}, \xi\right)-\left(\nabla \times\left(\boldsymbol{H}^{I}-\boldsymbol{H}\right), \xi\right)+\left(J\left(\boldsymbol{E}^{h}-\boldsymbol{E}\right), \xi\right), \\
\mu_{0}\left(\eta_{t}, \eta\right)+(\xi, \nabla \times \eta)=\mu_{0}\left(\left(\boldsymbol{H}^{I}-\boldsymbol{H}\right)_{t}, \eta\right)+\left(P_{h} \boldsymbol{E}-\boldsymbol{E}, \nabla \times \eta\right) .
\end{gathered}
$$

Adding the two equations above, we obtain

$$
\begin{aligned}
\frac{1}{2} \frac{d}{d t} & \left(\mu_{0}\|\eta(t)\|_{0}^{2}+\epsilon_{0}\|\xi(t)\|_{0}^{2}\right) \\
& =-\left(\nabla \times\left(\boldsymbol{H}^{I}-\boldsymbol{H}\right), \xi\right)+\left(J\left(\boldsymbol{E}^{h}-\boldsymbol{E}\right), \xi\right)+\mu_{0}\left(\left(\boldsymbol{H}^{I}-\boldsymbol{H}\right)_{t}, \eta\right) \\
& =\sum_{i=1}^{3}(E r r)_{i}
\end{aligned}
$$

where we used the definition of operator $P_{h}$ and the fact that $\nabla \times V_{h} \subset U_{h}$.

Below we will constantly use the basic arithmetic-geometric mean inequality

$$
|a b| \leq \delta a^{2}+\frac{1}{4 \delta} b^{2}
$$

for any constant $\delta>0$.

Now we will estimate $(E r r)_{i}$ one by one for $i=1,2,3$.

Using Lemma 2.1 and (23), we have

$$
\begin{aligned}
(E r r)_{1} & =-\left(\nabla \times\left(\boldsymbol{H}^{I}-\boldsymbol{H}\right), \xi\right) \leq C h^{k+1}\|\boldsymbol{H}(t)\|_{k+2}\|\xi(t)\|_{0} \\
& \leq \delta_{2} \epsilon_{0}\|\xi(t)\|_{0}^{2}+\frac{C_{1} h^{2(k+1)}}{4 \delta_{2} \epsilon_{0}}\|\boldsymbol{H}(t)\|_{k+2}^{2} .
\end{aligned}
$$

By the linearity of $J$ and the definition of $P_{h}$, we obtain

$$
\begin{aligned}
(E r r)_{2} & =\left(J\left(\boldsymbol{E}^{h}-P_{h} \boldsymbol{E}\right)+J\left(P_{h} \boldsymbol{E}-\boldsymbol{E}\right), \xi\right)=-(J(\xi), \xi) \\
& \leq \delta_{3} \epsilon_{0}\|\xi(t)\|_{0}^{2}+\frac{1}{4 \delta_{3} \epsilon_{0}}\|J(\xi)\|_{0}^{2} .
\end{aligned}
$$

Using the definition of $J$ and the Cauchy-Schwarz inequality, we have

$$
\begin{aligned}
\|J(\xi)\|_{0}^{2} & =\int_{\Omega}\left|\epsilon_{0} \omega_{p}^{2} \int_{0}^{t} e^{-\nu(t-s)} \xi(\mathbf{x}, s) d s\right|^{2} d \Omega \\
& \leq \epsilon_{0}^{2} \omega_{p}^{4} \int_{\Omega}\left(\int_{0}^{t}\left|e^{-\nu(t-s)}\right|^{2} d s\right)\left(\int_{0}^{t}|\xi(\mathbf{x}, s)|^{2} d s\right) d \Omega \\
& =\epsilon_{0}^{2} \omega_{p}^{4} \int_{\Omega} \frac{1}{2 \nu}\left(1-e^{-2 \nu t}\right)\left(\int_{0}^{t}|\xi(\mathbf{x}, s)|^{2} d s\right) d \Omega \\
& \leq \frac{\epsilon_{0}^{2} \omega_{p}^{4}}{2 \nu} \int_{0}^{t}\|\xi(s)\|_{0}^{2} d s
\end{aligned}
$$


which together with (24) gives

$$
(E r r)_{2} \leq \delta_{3} \epsilon_{0}\|\xi(t)\|_{0}^{2}+\frac{\epsilon_{0} \omega_{p}^{4}}{8 \delta_{3} \nu} \int_{0}^{t}\|\xi(s)\|_{0}^{2} d s .
$$

Using Lemma 2.2 and (23), we obtain

$$
\begin{aligned}
(E r r)_{3} & =\mu_{0}\left(\left(\boldsymbol{H}^{I}-\boldsymbol{H}\right)_{t}, \eta\right) \leq C \mu_{0} h^{k+1}\left\|\boldsymbol{H}_{t}(t)\right\|_{k+1}\|\eta(t)\|_{0} \\
& \leq \delta_{4} \mu_{0}\|\eta(t)\|_{0}^{2}+\frac{C_{2} \mu_{0} h^{2(k+1)}}{4 \delta_{4}}\left\|\boldsymbol{H}_{t}(t)\right\|_{k+1}^{2} .
\end{aligned}
$$

Combining all the estimates obtained for $(E r r)_{i}, i=1,2,3$, we shall have

$$
\begin{aligned}
& \frac{d}{d t}\left(\frac{\mu_{0}}{2}\|\eta(t)\|_{0}^{2}+\frac{\epsilon_{0}}{2}\|\xi(t)\|_{0}^{2}\right) \leq\left(\delta_{2}+\delta_{3}\right) \epsilon_{0}\|\xi(t)\|_{0}^{2}+\delta_{4} \mu_{0}\|\eta(t)\|_{0}^{2} \\
& +\frac{C_{1} h^{2(k+1)}}{4 \delta_{2} \epsilon_{0}}\|\boldsymbol{H}(t)\|_{k+2}^{2}+\frac{\epsilon_{0} \omega_{p}^{4}}{8 \delta_{3} \nu} \int_{0}^{t}\|\xi(s)\|_{0}^{2} d s+\frac{C_{2} \mu_{0} h^{2(k+1)}}{4 \delta_{4}}\left\|\boldsymbol{H}_{t}(t)\right\|_{k+1}^{2} .
\end{aligned}
$$

Integrating both sides of (26) with respect to $t$ and using the fact that $\xi(0)=$ $\eta(0)=0$, we obtain

$$
\begin{aligned}
& \mu_{0}\|\eta(t)\|_{0}^{2}+\epsilon_{0}\|\xi(t)\|_{0}^{2} \leq C_{3} \int_{0}^{t}\left(\mu_{0}\|\eta(s)\|_{0}^{2}+\epsilon_{0}\|\xi(s)\|_{0}^{2}\right) d s \\
& +C_{4} h^{2(k+1)} \int_{0}^{t}\left[\|\boldsymbol{H}(t)\|_{k+2}^{2}+\left\|\boldsymbol{H}_{t}(t)\right\|_{k+1}^{2}\right] d t
\end{aligned}
$$

where we denote

$$
C_{3}=2 \cdot \max \left\{\delta_{2}+\delta_{3}+\frac{t \omega_{p}^{4}}{8 \delta_{3} \nu}, \delta_{4}\right\}, \quad C_{4}=2 \cdot \max \left\{\frac{C_{1}}{4 \delta_{2} \epsilon_{0}}, \frac{C_{2} \mu_{0}}{4 \delta_{4}}\right\} .
$$

By the Gronwall's inequality (Lemma 2.3), we obtain

$$
\mu_{0}\|\eta(t)\|_{0}^{2}+\epsilon_{0}\|\xi(t)\|_{0}^{2} \leq C h^{2(k+1)} \int_{0}^{t}\left[\|\boldsymbol{H}(t)\|_{k+2}^{2}+\left\|\boldsymbol{H}_{t}(t)\right\|_{k+1}^{2}\right] d t,
$$

which completes the proof.

3.2. Debye medium. For the single pole model of Debye, the governing equations can be written as 22]: find $\boldsymbol{E}$ and $\mathbf{H}$, which satisfy

$$
\begin{aligned}
\epsilon_{0} \epsilon_{\infty} \boldsymbol{E}_{t}-\nabla \times \boldsymbol{H}+\frac{\left(\epsilon_{s}-\epsilon_{\infty}\right) \epsilon_{0}}{t_{0}} \boldsymbol{E}-\tilde{\boldsymbol{J}}(\boldsymbol{E})=0 & \text { in } \Omega \times(0, T), \\
\mu_{0} \boldsymbol{H}_{t}+\nabla \times \boldsymbol{E} & =0 \quad \text { in } \Omega \times(0, T),
\end{aligned}
$$

with the same boundary and initial conditions as those stated previously for plasma. Here we introduce the pseudo polarization current

$$
\tilde{\boldsymbol{J}}(\boldsymbol{E}) \equiv \tilde{\boldsymbol{J}}(\mathbf{x}, t ; \boldsymbol{E})=\frac{\left(\epsilon_{s}-\epsilon_{\infty}\right) \epsilon_{0}}{t_{0}^{2}} \int_{0}^{t} e^{-\frac{(t-s)}{t_{0}}} \boldsymbol{E}(\mathbf{x}, s) d s
$$

in order to carry out our earlier analysis for plasma easily to the Debye medium. Furthermore, $\epsilon_{\infty}$ is the permittivity at infinite frequency, $\epsilon_{s}$ is the permittivity at zero frequency, $t_{0}$ is the relaxation time, and the rest have the same meaning as those stated previously for the plasma model. 
From (29)-(30), we can easily obtain the weak formulation: find the solution $(\boldsymbol{E}, \boldsymbol{H}) \in\left[C^{1}\left(0, T ;\left(L^{2}(\Omega)\right)^{3}\right) \cap C^{0}(0, T ; H(\operatorname{curl} ; \Omega))\right]^{2}$ such that

$$
\begin{aligned}
\epsilon_{0} \epsilon_{\infty}\left(\boldsymbol{E}_{t}, \boldsymbol{\phi}\right)-(\nabla \times \boldsymbol{H}, \boldsymbol{\phi}) & \\
+\frac{\left(\epsilon_{s}-\epsilon_{\infty}\right) \epsilon_{0}}{t_{0}}(\boldsymbol{E}, \boldsymbol{\phi})-(\tilde{\boldsymbol{J}}(\boldsymbol{E}), \boldsymbol{\phi})=0 & \forall \boldsymbol{\phi} \in\left(L^{2}(\Omega)\right)^{3}, \\
\mu_{0}\left(\boldsymbol{H}_{t}, \boldsymbol{\psi}\right)+(\boldsymbol{E}, \nabla \times \boldsymbol{\psi})=0 & \forall \boldsymbol{\psi} \in H(\operatorname{curl} ; \Omega)
\end{aligned}
$$

for $0<t \leq T$ with the initial conditions

$$
\boldsymbol{E}(\boldsymbol{x}, 0)=\boldsymbol{E}_{0}(\boldsymbol{x}) \text { and } \boldsymbol{H}(\boldsymbol{x}, 0)=\boldsymbol{H}_{0}(\boldsymbol{x}) .
$$

The semi-discrete mixed finite element scheme for our Debye model can be formulated as follows: $\left(\boldsymbol{E}^{h}, \boldsymbol{H}^{h}\right) \in C^{1}\left(0, T ; \mathbf{U}_{h}\right) \times C^{1}\left(0, T ; \mathbf{V}_{h}\right)$ such that

$$
\begin{aligned}
\epsilon_{0} \epsilon_{\infty}\left(\boldsymbol{E}_{t}^{h}, \boldsymbol{\phi}_{h}\right)-\left(\nabla \times \boldsymbol{H}^{h}, \boldsymbol{\phi}_{h}\right) & \\
+\frac{\left(\epsilon_{s}-\epsilon_{\infty}\right) \epsilon_{0}}{t_{0}}\left(\boldsymbol{E}^{h}, \boldsymbol{\phi}_{h}\right)-\left(\tilde{\boldsymbol{J}}\left(\boldsymbol{E}^{h}\right), \boldsymbol{\phi}_{h}\right)=0 & \forall \boldsymbol{\phi}_{h} \in \mathbf{U}_{h}, \\
\mu_{0}\left(\boldsymbol{H}_{t}^{h}, \boldsymbol{\psi}_{h}\right)+\left(\boldsymbol{E}^{h}, \nabla \times \boldsymbol{\psi}_{h}\right)=0 & \forall \boldsymbol{\psi}_{h} \in \mathbf{V}_{h}
\end{aligned}
$$

for $0<t \leq T$, subject to the initial conditions

$$
\boldsymbol{E}^{h}(\boldsymbol{x}, 0)=P_{h} \boldsymbol{E}_{0}(\boldsymbol{x}) \quad \text { and } \quad \boldsymbol{H}^{h}(\boldsymbol{x}, 0)=\boldsymbol{H}_{0}^{I}(\boldsymbol{x}) .
$$

Theorem 3.2. Let $(\boldsymbol{E}(t), \boldsymbol{H}(t))$ and $\left(\boldsymbol{E}^{h}(t), \boldsymbol{H}^{h}(t)\right)$ be the solutions of (32)-(33) and (35) -(36) at time $t$, respectively. Then there is a constant $C=C\left(\epsilon_{0}, \mu_{0}, \epsilon_{\infty}, \epsilon_{s}, t_{0}\right)$, independent of the mesh size $h$, such that

$$
\begin{aligned}
\mu_{0} \|\left(\boldsymbol{H}^{I}-\boldsymbol{H}^{h}\right) & (t)\left\|_{0}^{2}+\epsilon_{0} \epsilon_{\infty}\right\|\left(P_{h} \boldsymbol{E}-\boldsymbol{E}^{h}\right)(t) \|_{0}^{2} \\
& \leq C h^{2(k+1)} \int_{0}^{t}\left[\|\boldsymbol{H}(t)\|_{k+2}^{2}+\left\|\boldsymbol{H}_{t}(t)\right\|_{k+1}^{2}\right] d t,
\end{aligned}
$$

where $k$ is the degree of edge elements in the space $\boldsymbol{V}_{h}$.

Proof. Subtracting (35)-(36) from (32)-(33) gives the error equations:

$$
\begin{aligned}
& \epsilon_{0} \epsilon_{\infty}\left(\left(\boldsymbol{E}-\boldsymbol{E}^{h}\right)_{t}, \boldsymbol{\phi}_{h}\right)-\left(\nabla \times\left(\boldsymbol{H}-\boldsymbol{H}^{h}\right), \boldsymbol{\phi}_{h}\right)+\frac{\left(\epsilon_{s}-\epsilon_{\infty}\right) \epsilon_{0}}{t_{0}}\left(\boldsymbol{E}-\boldsymbol{E}^{h}, \boldsymbol{\phi}_{h}\right) \\
& \quad-\left(\tilde{\boldsymbol{J}}\left(\boldsymbol{E}-\boldsymbol{E}^{h}\right), \boldsymbol{\phi}_{h}\right)=0 \quad \forall \boldsymbol{\phi}_{h} \in \mathbf{U}_{h}, \\
& \mu_{0}\left(\left(\boldsymbol{H}-\boldsymbol{H}^{h}\right)_{t}, \boldsymbol{\psi}_{h}\right)+\left(\boldsymbol{E}-\boldsymbol{E}^{h}, \nabla \times \boldsymbol{\psi}_{h}\right)=0 \quad \forall \boldsymbol{\psi}_{h} \in \mathbf{V}_{h} .
\end{aligned}
$$

Choosing $\phi_{h}=\xi, \psi_{h}=\eta$ in (39)-(40), and rearranging terms lead to

$$
\begin{gathered}
\epsilon_{0} \epsilon_{\infty}\left(\xi_{t}, \xi\right)-(\nabla \times \eta, \xi)+\frac{\left(\epsilon_{s}-\epsilon_{\infty}\right) \epsilon_{0}}{t_{0}}(\xi, \xi)=\epsilon_{0} \epsilon_{\infty}\left(\left(P_{h} \boldsymbol{E}-\boldsymbol{E}\right)_{t}, \xi\right) \\
-\left(\nabla \times\left(\boldsymbol{H}^{I}-\boldsymbol{H}\right), \xi\right)+\frac{\left(\epsilon_{s}-\epsilon_{\infty}\right) \epsilon_{0}}{t_{0}}\left(P_{h} \boldsymbol{E}-\boldsymbol{E}, \xi\right)+\left(\tilde{\boldsymbol{J}}\left(\boldsymbol{E}-\boldsymbol{E}^{h}\right), \xi\right), \\
\mu_{0}\left(\eta_{t}, \eta\right)+(\xi, \nabla \times \eta)=\mu_{0}\left(\left(\boldsymbol{H}^{I}-\boldsymbol{H}\right)_{t}, \eta\right)+\left(P_{h} \boldsymbol{E}-\boldsymbol{E}, \nabla \times \eta\right) .
\end{gathered}
$$


Adding the above two equations together and using the definition of $P_{h}$, we obtain

$$
\begin{aligned}
& \frac{d}{d t}\left(\frac{\epsilon_{0} \epsilon_{\infty}}{2}\|\xi(t)\|_{0}^{2}+\frac{\mu_{0}}{2}\|\eta(t)\|_{0}^{2}\right)+\frac{\left(\epsilon_{s}-\epsilon_{\infty}\right) \epsilon_{0}}{t_{0}}\|\xi(t)\|_{0}^{2} \\
& \quad=-\left(\nabla \times\left(\boldsymbol{H}^{I}-\boldsymbol{H}\right), \xi\right)+\left(\tilde{\boldsymbol{J}}\left(\boldsymbol{E}-\boldsymbol{E}^{h}\right), \xi\right)+\mu_{0}\left(\left(\boldsymbol{H}^{I}-\boldsymbol{H}\right)_{t}, \eta\right) \\
& \quad=\sum_{i=1}^{3}(E r r)_{i} .
\end{aligned}
$$

The rest of the proof is omitted because it is very similar to the plasma case.

3.3. Lorentz medium. The Lorentzian two pole model can be represented by the governing equations $[22$ :

$$
\begin{array}{rlll}
\epsilon_{0} \epsilon_{\infty} \boldsymbol{E}_{t}-\nabla \times \boldsymbol{H}+\hat{\boldsymbol{J}}(\boldsymbol{E}) & =0 & \text { in } \Omega \times(0, T), \\
\mu_{0} \boldsymbol{H}_{t}+\nabla \times \boldsymbol{E} & =0 & \text { in } \Omega \times(0, T),
\end{array}
$$

with the same boundary and initial conditions as those stated previously for plasma. We use $\hat{\boldsymbol{J}}$ to represent the polarization current for the Lorentz medium:

$$
\begin{aligned}
\hat{\boldsymbol{J}}(\boldsymbol{E}) & \equiv \hat{\boldsymbol{J}}(\mathbf{x}, t ; \boldsymbol{E})=\tilde{\beta} \int_{0}^{t} e^{-\delta(t-s)} \cdot \sin (\gamma-\alpha(t-s)) \cdot \boldsymbol{E}(\mathbf{x}, s) d s, \\
& =\operatorname{Im}\left(\tilde{\beta} e^{j \gamma} \int_{0}^{t} e^{-(\delta+j \alpha)(t-s)} \boldsymbol{E}(\mathbf{x}, s) d s\right) \equiv \operatorname{Im}(\overline{\boldsymbol{J}}(\boldsymbol{E})), j=\sqrt{-1},
\end{aligned}
$$

where $\tilde{\beta}=\left(\epsilon_{s}-\epsilon_{\infty}\right) \epsilon_{0} \omega_{1}^{3} / \sqrt{\omega_{1}^{2}-\frac{\nu^{2}}{4}}$, and $\operatorname{Im}(A)$ denotes the imaginary part of a general complex number $A$. Furthermore, in addition to the notation defined earlier, $\omega_{1}$ is the resonant frequency, $\nu$ is the damping coefficient, $\delta=\frac{\nu}{2}, \alpha=\sqrt{\omega_{1}^{2}-\delta^{2}}$ and $e^{j \gamma}=\frac{\delta}{\omega_{1}}+j \frac{\alpha}{\omega_{1}}$. Note that $\omega_{1}>\delta$ in real applications [17.

From (42)- (43), we can easily obtain the weak formulation: find the solution $(\boldsymbol{E}, \boldsymbol{H}) \in\left[C^{1}\left(0, T ;\left(L^{2}(\Omega)\right)^{3}\right) \cap C^{0}(0, T ; H(\operatorname{curl} ; \Omega))\right]^{2}$ such that

$$
\begin{aligned}
& \epsilon_{0} \epsilon_{\infty}\left(\boldsymbol{E}_{t}, \boldsymbol{\phi}\right)-(\nabla \times \boldsymbol{H}, \boldsymbol{\phi})+(\hat{\boldsymbol{J}}(\boldsymbol{E}), \boldsymbol{\phi})=0 \quad \forall \boldsymbol{\phi} \in\left(L^{2}(\Omega)\right)^{3}, \\
& \mu_{0}\left(\boldsymbol{H}_{t}, \boldsymbol{\psi}\right)+(\boldsymbol{E}, \nabla \times \boldsymbol{\psi})=0 \quad \forall \boldsymbol{\psi} \in H(\operatorname{curl} ; \Omega)
\end{aligned}
$$

for $0<t \leq T$ with the initial conditions

$$
\boldsymbol{E}(\boldsymbol{x}, 0)=\boldsymbol{E}_{0}(\boldsymbol{x}) \text { and } \boldsymbol{H}(\boldsymbol{x}, 0)=\boldsymbol{H}_{0}(\boldsymbol{x}) .
$$

The semi-discrete mixed finite element scheme for the Lorentz medium is formulated as follows: find $\boldsymbol{E}^{h} \in \mathbf{U}_{h}, \boldsymbol{H}^{h} \in \mathbf{V}_{h}$ such that

$$
\begin{aligned}
\epsilon_{0} \epsilon_{\infty}\left(\boldsymbol{E}_{t}^{h}, \phi_{h}\right)-\left(\nabla \times \boldsymbol{H}^{h}, \phi_{h}\right)+\left(\hat{\boldsymbol{J}}\left(\boldsymbol{E}^{h}\right), \phi_{h}\right) & =0 \quad \forall \phi_{h} \in \mathbf{U}_{h}, \\
\mu_{0}\left(\boldsymbol{H}_{t}^{h}, \boldsymbol{\psi}_{h}\right)+\left(\boldsymbol{E}^{h}, \nabla \times \boldsymbol{\psi}_{h}\right) & =0 \quad \forall \boldsymbol{\psi}_{h} \in \mathbf{V}_{h}
\end{aligned}
$$

for $0<t \leq T$, subject to the initial conditions

$$
\boldsymbol{E}^{h}(\boldsymbol{x}, 0)=P_{h} \boldsymbol{E}_{0}(\boldsymbol{x}) \text { and } \boldsymbol{H}^{h}(\boldsymbol{x}, 0)=\boldsymbol{H}_{0}^{I}(\boldsymbol{x}) .
$$

Theorem 3.3. Let $(\boldsymbol{E}(t), \boldsymbol{H}(t))$ and $\left(\boldsymbol{E}^{h}(t), \boldsymbol{H}^{h}(t)\right)$ be the solutions of (46) -(47) and (49)-(50) at time $t$, respectively. Then there is a constant

$$
C=C\left(\epsilon_{0}, \mu_{0}, \epsilon_{s}, \epsilon_{\infty}, \omega_{1}, \nu\right)
$$


independent of the mesh size $h$, such that

$$
\begin{aligned}
& \mu_{0} \|\left(\boldsymbol{H}^{I}-\boldsymbol{H}^{h}\right)(t)\left\|_{0}^{2}+\epsilon_{0} \epsilon_{\infty}\right\|\left(P_{h} \boldsymbol{E}-\boldsymbol{E}^{h}\right)(t) \|_{0}^{2} \\
& \leq C h^{2(k+1)} \int_{0}^{t}\left[\|\boldsymbol{H}(t)\|_{k+2}^{2}+\left\|\boldsymbol{H}_{t}(t)\right\|_{k+1}^{2}\right] d t
\end{aligned}
$$

where $k$ is the degree of edge elements in the space $\boldsymbol{V}_{h}$.

Proof. Subtracting (49)-(50) from (46)-(47) with $\phi=\phi_{h}=\xi(t)$ and $\boldsymbol{\psi}=\boldsymbol{\psi}_{h}=$ $\eta(t)$, respectively, we can obtain the error equations:

$$
\begin{aligned}
\epsilon_{0} \epsilon_{\infty}\left(\xi_{t}, \xi\right)-(\nabla \times \eta, \xi)= & \epsilon_{0} \epsilon_{\infty}\left(\left(P_{h} \boldsymbol{E}-\boldsymbol{E}\right)_{t}, \xi\right) \\
& -\left(\nabla \times\left(\boldsymbol{H}^{I}-\boldsymbol{H}\right), \xi\right)-\left(\hat{\boldsymbol{J}}\left(\boldsymbol{E}-\boldsymbol{E}^{h}\right), \xi\right), \\
\mu_{0}\left(\eta_{t}, \eta\right)+(\xi, \nabla \times \eta)= & \mu_{0}\left(\left(\boldsymbol{H}^{I}-\boldsymbol{H}\right)_{t}, \eta\right)+\left(P_{h} \boldsymbol{E}-\boldsymbol{E}, \nabla \times \eta\right) .
\end{aligned}
$$

Adding the above two equations and using the definition of $P_{h}$, we obtain

$$
\begin{aligned}
& \frac{d}{d t}\left(\frac{\mu_{0}}{2}\|\eta(t)\|_{0}^{2}+\frac{\epsilon_{0} \epsilon_{\infty}}{2}\|\xi(t)\|_{0}^{2}\right) \\
& \quad=-\left(\nabla \times\left(\boldsymbol{H}^{I}-\boldsymbol{H}\right), \xi\right)-\left(\hat{\boldsymbol{J}}\left(\boldsymbol{E}-\boldsymbol{E}^{h}\right), \xi\right)+\mu_{0}\left(\left(\boldsymbol{H}^{I}-\boldsymbol{H}\right)_{t}, \eta\right) \\
& \quad=\sum_{i=1}^{3}(E r r)_{i} .
\end{aligned}
$$

The estimates of $(E r r)_{i}$ follow the proof for the plasma case. The only different term is $(E r r)_{2}$.

By the linearity of $\hat{\boldsymbol{J}}$ and the definition of $P_{h}$, we obtain

$$
\begin{aligned}
(E r r)_{2} & =-\left(\hat{\boldsymbol{J}}\left(\boldsymbol{E}-P_{h} \boldsymbol{E}\right)+\boldsymbol{J}\left(P_{h} \boldsymbol{E}-\boldsymbol{E}^{h}\right), \xi\right)=-(\hat{\boldsymbol{J}}(\xi), \xi) \\
& \leq \delta_{3} \epsilon_{0} \epsilon_{\infty}\|\xi(t)\|_{0}^{2}+\frac{1}{4 \delta_{3} \epsilon_{0} \epsilon_{\infty}}\|\hat{\boldsymbol{J}}(\xi)\|_{0}^{2}
\end{aligned}
$$

It is easy to see that

$$
\begin{aligned}
\|\hat{\boldsymbol{J}}(\xi)\|_{0}^{2} \leq\|\overline{\boldsymbol{J}}(\xi)\|_{0}^{2} & \left.=\tilde{\beta}^{2} \int_{\Omega}\left|e^{j \gamma} \int_{0}^{t} e^{-(\delta+j \alpha)(t-s)} \xi(\mathbf{x}, s) d s\right|^{2} d \Omega\right) \\
& \leq \tilde{\beta}^{2} \frac{1}{2 \delta} \int_{0}^{t}\|\xi(s)\|_{0}^{2} d s
\end{aligned}
$$

which together with (54) gives

$$
(E r r)_{2} \leq \delta_{3} \epsilon_{0} \epsilon_{\infty}\|\xi(t)\|_{0}^{2}+\frac{\tilde{\beta}^{2}}{8 \delta_{3} \epsilon_{0} \epsilon_{\infty} \delta} \int_{0}^{t}\|\xi(s)\|_{0}^{2} d s .
$$

The rest of the proof follows exactly the same as the plasma case.

\section{Global superconvergence}

To prove global superconvergence, we need some postprocessing operators introduced by Lin and Yan [29].

For each component $w_{j}, j=1,2,3$ of $\mathbf{w} \in \mathbf{U}_{h}$, we define $\left.\Pi_{2 h} w_{j}\right|_{\hat{e}} \in Q_{k, 2 k-1,2 k-1}(\hat{e})$ such that

$$
\int_{e_{i}}\left(\Pi_{2 h} w_{j}-w_{j}\right) q=0, \quad \forall q \in Q_{k, k-1, k-1}\left(e_{i}\right), i=1,2,3,4,
$$

where $\hat{e}=\sum_{i=1}^{4} e_{i}$. 
Another postprocessing operator $\hat{\Pi}_{2 h}$ is defined for the function $\mathbf{v} \in \mathbf{V}_{h}$. For the first component $v_{1}$ of $\mathbf{v}$, we define $\left.\hat{\Pi}_{2 h} v_{1}\right|_{\check{e}} \in Q_{2 k-1, k, k}(\check{e})$ such that

$$
\begin{aligned}
& \int_{l_{i}}\left(\hat{\Pi}_{2 h} v_{1}-v_{1}\right) q d x=0, \quad \forall q \in P_{k-1}\left(l_{i}\right), i=1, \cdots, 8, \\
& \int_{\tau_{i}}\left(\hat{\Pi}_{2 h} v_{1}-v_{1}\right) q d x d y=0, \quad \forall q \in Q_{k-1, k-2}\left(\tau_{i}\right), i=1, \cdots, 4, \\
& \int_{\tau_{j}}\left(\hat{\Pi}_{2 h} v_{1}-v_{1}\right) q d x d z=0, \quad \forall q \in Q_{k-1, k-2}\left(\tau_{j}\right), j=1, \cdots, 4, \\
& \int_{e_{i}}\left(\hat{\Pi}_{2 h} v_{1}-v_{1}\right) q d x d y d z=0, \quad \forall q \in Q_{k-1, k-2, k-2}\left(e_{i}\right), i=1,2,
\end{aligned}
$$

where $\check{e}=e_{1} \cup e_{2}, l_{i}$ are edges parallel to the $x$-axis, $\tau_{i}, \tau_{j}$ are surfaces perpendicular to the $z$-axis or $y$-axis, respectively. $\hat{\Pi}_{2 h}$ can be defined similarly for the second and third components of $\mathbf{v} \in \mathbf{V}_{h}$.

Lin and Yan 29] proved the following properties:

\section{Lemma 4.1.}

(i) $\left\|\Pi_{2 h} \mathbf{w}-\mathbf{w}\right\|_{0} \leq C h^{k+1}\|\mathbf{w}\|_{k+1}, \quad\left\|\hat{\Pi}_{2 h} \mathbf{v}-\mathbf{v}\right\|_{0} \leq C h^{k+1}\|\mathbf{v}\|_{k+1}$, $\forall \mathbf{w}, \mathbf{v} \in\left(H^{k+1}(\Omega)\right)^{3}$,

(ii) $\left\|\Pi_{2 h} \mathbf{w}\right\|_{0} \leq C\|\mathbf{w}\|_{0},\left\|\hat{\Pi}_{2 h} \mathbf{v}\right\|_{0} \leq C\|\mathbf{v}\|_{0}, \quad \forall \mathbf{w} \in \mathbf{U}_{h}, \mathbf{v} \in \mathbf{V}_{h}$,

(iii) $\Pi_{2 h} \mathbf{w}=\Pi_{2 h} P_{h} \mathbf{w}, \hat{\Pi}_{2 h} \mathbf{v}=\hat{\Pi}_{2 h} \mathbf{v}^{\mathbf{I}}, \quad \forall \mathbf{w} \in \mathbf{U}_{h}, \mathbf{v} \in \mathbf{V}_{h}$, where $P_{h} \mathbf{w} \in \mathbf{U}_{h}$ and $\mathbf{v}^{\mathbf{I}} \in \mathbf{V}_{h}$ are the interpolations of $\mathbf{w}$ and $\mathbf{v}$ defined in Section 2.

Using these postprocessing operators, we can achieve the following global superconvergence for all three dispersive media:

\section{Theorem 4.1.}

$$
\begin{aligned}
& \left\|\Pi_{2 h} \boldsymbol{E}^{h}-\boldsymbol{E}\right\|_{0}+\left\|\hat{\Pi}_{2 h} \boldsymbol{H}^{h}-\boldsymbol{H}\right\|_{0} \\
& \quad \leq C h^{k+1}\left[\|\boldsymbol{E}\|_{k+1}+\|\boldsymbol{H}\|_{k+1}+\left(\int_{0}^{t}\left(\|\boldsymbol{H}\|_{k+2}^{2}+\left\|\boldsymbol{H}_{t}\right\|_{k+1}^{2}\right) d s\right)^{1 / 2}\right],
\end{aligned}
$$

where $k$ is the degree of edge elements in the space $\boldsymbol{V}_{h}$.

Proof. By Lemma 4.1 and Theorems 3.13 .3 we have

$$
\begin{aligned}
\left\|\Pi_{2 h} \boldsymbol{E}^{h}-\boldsymbol{E}\right\|_{0} & =\left\|\Pi_{2 h}\left(\boldsymbol{E}^{h}-P_{h} \boldsymbol{E}\right)+\left(\Pi_{2 h} \boldsymbol{E}-\boldsymbol{E}\right)\right\|_{0} \\
& \leq C\left\|\boldsymbol{E}^{h}-P_{h} \boldsymbol{E}\right\|_{0}+C h^{k+1}\|\boldsymbol{E}\|_{k+1} \\
& \leq C h^{k+1}\left[\left(\int_{0}^{t}\left(\|\boldsymbol{H}\|_{k+2}^{2}+\left\|\boldsymbol{H}_{t}\right\|_{k+1}^{2}\right) d s\right)^{1 / 2}+\|\boldsymbol{E}\|_{k+1}\right] .
\end{aligned}
$$

Similarly, we have

$$
\begin{aligned}
\left\|\hat{\Pi}_{2 h} \boldsymbol{H}^{h}-\boldsymbol{H}\right\|_{0} & =\left\|\hat{\Pi}_{2 h}\left(\boldsymbol{H}^{h}-\boldsymbol{H}^{I}\right)+\left(\hat{\Pi}_{2 h} \boldsymbol{H}-\boldsymbol{H}\right)\right\|_{0} \\
& \leq C\left\|\boldsymbol{H}^{h}-\boldsymbol{H}^{I}\right\|_{0}+C h^{k+1}\|\boldsymbol{H}\|_{k+1} \\
& \leq C h^{k+1}\left[\left(\int_{0}^{t}\left(\|\boldsymbol{H}\|_{k+2}^{2}+\left\|\boldsymbol{H}_{t}\right\|_{k+1}^{2}\right) d s\right)^{1 / 2}+\|\boldsymbol{H}\|_{k+1}\right]
\end{aligned}
$$

which, along with (55), completes the proof. 


\section{EXTENSION TO A STANDARD FINITE ELEMENT METHOD}

In this section, we will extend the global superconvergence to a standard finite element method. For simplicity, we will restrict our discussion to the cold plasma model, generalizations to other models are similar.

Instead of solving the coupled system (5)-(7) with both the electric and magnetic fields as unknowns, we eliminate $\boldsymbol{H}$ by taking the time derivative of (5) and using (6) -(8), to obtain the second order electric field equation

$$
\epsilon_{0} \boldsymbol{E}_{t t}+\nabla \times\left(\mu_{0}^{-1} \nabla \times \boldsymbol{E}\right)+\epsilon_{0} \omega_{p}^{2} \boldsymbol{E}-\nu \boldsymbol{J}(\boldsymbol{E})=0,
$$

with boundary condition (11) and initial conditions

$$
\boldsymbol{E}(\boldsymbol{x}, 0)=\boldsymbol{E}_{0}(\boldsymbol{x}) \quad \text { and } \quad \boldsymbol{E}_{t}(\boldsymbol{x}, 0)=\boldsymbol{E}_{1}(\boldsymbol{x}),
$$

where $\boldsymbol{E}_{1}(\boldsymbol{x})=\epsilon_{0}^{-1} \nabla \times \boldsymbol{H}_{0}(\boldsymbol{x})$, which is obtained from (5), (8), and (12).

Multiplying (57) by $\phi \in H_{0}(\operatorname{curl} ; \Omega)$, and using integration by parts [35. (3.27)], we can easily obtain the weak formulation: find $\boldsymbol{E}(t) \in H_{0}(\operatorname{curl} ; \Omega)$ such that

$$
\begin{gathered}
\epsilon_{0}\left(\boldsymbol{E}_{t t}, \boldsymbol{\phi}\right)+\mu_{0}^{-1}(\nabla \times \boldsymbol{E}, \nabla \times \boldsymbol{\phi})+\epsilon_{0} \omega_{p}^{2}(\boldsymbol{E}, \boldsymbol{\phi}) \\
-\nu(\boldsymbol{J}(\boldsymbol{E}), \boldsymbol{\phi})=0 \quad \boldsymbol{\phi} \in H_{0}(\operatorname{curl} ; \Omega),
\end{gathered}
$$

subject to the initial conditions (58).

Taking the boundary condition (11) into account, we define

$$
\boldsymbol{V}_{h}^{0}=\left\{\boldsymbol{v}_{h} \in \boldsymbol{V}_{h} \mid \mathbf{n} \times \boldsymbol{v}_{h}=0 \text { on } \partial \Omega\right\} .
$$

Then we can formulate a standard finite element scheme for (57) as follows: find $\boldsymbol{E}^{h}(t) \in \boldsymbol{V}_{h}^{0}$ such that

(60) $\epsilon_{0}\left(\boldsymbol{E}_{t t}^{h}, \boldsymbol{\phi}\right)+\mu_{0}^{-1}\left(\nabla \times \boldsymbol{E}^{h}, \nabla \times \boldsymbol{\phi}\right)+\epsilon_{0} \omega_{p}^{2}\left(\boldsymbol{E}^{h}, \boldsymbol{\phi}\right)-\nu\left(\boldsymbol{J}\left(\boldsymbol{E}^{h}\right), \boldsymbol{\phi}\right)=0 \quad \forall \boldsymbol{\phi} \in \boldsymbol{V}_{h}^{0}$,

subject to the initial conditions

$$
\boldsymbol{E}^{h}(\boldsymbol{x}, 0)=\boldsymbol{E}_{0}^{I}(\boldsymbol{x}), \quad \boldsymbol{E}_{t}^{h}(\boldsymbol{x}, 0)=\boldsymbol{E}_{1}^{I}(\boldsymbol{x}),
$$

where $\boldsymbol{E}_{0}^{I}$ and $\boldsymbol{E}_{1}^{I}$ are the interpolations of $\boldsymbol{E}_{0}$ and $\boldsymbol{E}_{1}$ defined in $\S 2$, respectively.

Theorem 5.1. Let $\boldsymbol{E}(t)$ and $\boldsymbol{E}^{h}(t)$ be the solutions of (59) and (60) at time $t$, respectively. Then there is a constant $C=C\left(\epsilon_{0}, \mu_{0}, \omega_{p}, \nu\right)$, independent of the mesh size $h$, such that

$$
\begin{gathered}
\epsilon_{0}\left\|\left(\boldsymbol{E}^{I}-\boldsymbol{E}^{h}\right)_{t}(t)\right\|_{0}^{2}+\mu_{0}^{-1}\left\|\nabla \times\left(\boldsymbol{E}^{I}-\boldsymbol{E}^{h}\right)(t)\right\|_{0}^{2}+\epsilon_{0} \omega_{p}^{2}\left\|\left(\boldsymbol{E}^{I}-\boldsymbol{E}^{h}\right)(t)\right\|_{0}^{2} \\
\leq C h^{2(k+1)}\left[\|\boldsymbol{E}\|_{k+2}^{2}+\int_{0}^{t}\left(\left\|\boldsymbol{E}_{t t}\right\|_{k+1}^{2}+\left\|\boldsymbol{E}_{t}\right\|_{k+2}^{2}+\|\boldsymbol{E}\|_{k+1}^{2}\right) d s\right] .
\end{gathered}
$$

Proof. Denote $\xi(t)=\left(\boldsymbol{E}^{I}-\boldsymbol{E}^{h}\right)(t)$. Subtracting (60) from (59) with $\boldsymbol{\phi}=\xi_{t}(t)$, and rearranging terms, we obtain the error equation

$$
\begin{aligned}
\epsilon_{0}\left(\xi_{t t}, \xi_{t}\right)+\mu_{0}^{-1}\left(\nabla \times \xi, \nabla \times \xi_{t}\right)+\epsilon_{0} \omega_{p}^{2}\left(\xi, \xi_{t}\right) \\
=\epsilon_{0}\left(\left(\boldsymbol{E}^{I}-\boldsymbol{E}\right)_{t t}, \xi_{t}\right)+\mu_{0}^{-1}\left(\nabla \times\left(\boldsymbol{E}^{I}-\boldsymbol{E}\right), \nabla \times \xi_{t}\right) \\
\quad+\epsilon_{0} \omega_{p}^{2}\left(\boldsymbol{E}^{I}-\boldsymbol{E}, \xi_{t}\right)+\nu\left(\boldsymbol{J}\left(\boldsymbol{E}-\boldsymbol{E}^{I}\right), \xi_{t}\right)+\nu\left(\boldsymbol{J}(\xi), \xi_{t}\right) .
\end{aligned}
$$


Integrating (62) with respect to $t$ and using integration by parts and the fact that $\xi(0)=\xi_{t}(0)=0$, we obtain

$$
\begin{aligned}
& \frac{1}{2}\left(\epsilon_{0}\left\|\xi_{t}\right\|_{0}^{2}+\mu_{0}^{-1}\|\nabla \times \xi\|_{0}^{2}+\epsilon_{0} \omega_{p}^{2}\|\xi\|_{0}^{2}\right) \\
& \leq \epsilon_{0} \int_{0}^{t}\left(\left(\boldsymbol{E}^{I}-\boldsymbol{E}\right)_{t t}, \xi_{t}\right) d s+\mu_{0}^{-1}\left(\nabla \times\left(\boldsymbol{E}^{I}-\boldsymbol{E}\right), \nabla \times \xi\right) \\
& \quad-\mu_{0}^{-1} \int_{0}^{t}\left(\nabla \times\left(\boldsymbol{E}^{I}-\boldsymbol{E}\right)_{t}, \nabla \times \xi\right) d s \\
& \quad+\epsilon_{0} \omega_{p}^{2}\left(\boldsymbol{E}^{I}-\boldsymbol{E}, \xi\right)-\epsilon_{0} \omega_{p}^{2} \int_{0}^{t}\left(\left(\boldsymbol{E}^{I}-\boldsymbol{E}\right)_{t}, \xi\right) d s \\
& \quad+\nu \int_{0}^{t}\left(\boldsymbol{J}\left(\boldsymbol{E}-\boldsymbol{E}^{I}\right), \xi_{t}\right) d s+\nu \int_{0}^{t}\left(\boldsymbol{J}(\xi), \xi_{t}\right) d s=\sum_{i=1}^{7}(E r r)_{i} .
\end{aligned}
$$

Now we have to estimate all $(E r r)_{i}, i=1, \cdots, 7$.

Using Lemma 2.2 and the inequality (23), we have

$$
\begin{aligned}
(E r r)_{1} & =\epsilon_{0} \int_{0}^{t}\left(\left(\boldsymbol{E}^{I}-\boldsymbol{E}\right)_{t t}, \xi_{t}\right) d s \leq \epsilon_{0} \int_{0}^{t} C h^{k+1}\left\|\boldsymbol{E}_{t t}\right\|_{k+1}\left\|\xi_{t}\right\|_{0} d s \\
& \leq \int_{0}^{t} \delta_{1} \epsilon_{0}\left\|\xi_{t}\right\|_{0}^{2} d s+\int_{0}^{t} \frac{C \epsilon_{0} h^{2(k+1)}}{4 \delta_{1}}\left\|\boldsymbol{E}_{t t}\right\|_{k+1}^{2} d s .
\end{aligned}
$$

Using Lemma 2.1 and the inequality (23) leads to

$$
\begin{aligned}
(E r r)_{2} & =\mu_{0}^{-1}\left(\nabla \times\left(\boldsymbol{E}^{I}-\boldsymbol{E}\right), \nabla \times \xi\right) \leq \mu_{0}^{-1} C h^{k+1}\|\boldsymbol{E}\|_{k+2}\|\nabla \times \xi\|_{0} \\
& \leq \delta_{2} \mu_{0}^{-1}\|\nabla \times \xi\|_{0}^{2}+\frac{C h^{2(k+1)}}{4 \delta_{2} \mu_{0}}\|\boldsymbol{E}\|_{k+2}^{2}
\end{aligned}
$$

and

$$
\begin{aligned}
(E r r)_{3} & =-\mu_{0}^{-1} \int_{0}^{t}\left(\nabla \times\left(\boldsymbol{E}^{I}-\boldsymbol{E}\right)_{t}, \nabla \times \xi\right) d s \leq \mu_{0}^{-1} \int_{0}^{t} C h^{k+1}\left\|\boldsymbol{E}_{t}\right\|_{k+2}\|\nabla \times \xi\|_{0} d s \\
& \leq \int_{0}^{t} \delta_{3} \mu_{0}^{-1}\|\nabla \times \xi\|_{0}^{2} d s+\frac{C h^{2(k+1)}}{4 \delta_{3} \mu_{0}} \int_{0}^{t}\left\|\boldsymbol{E}_{t}\right\|_{k+2}^{2} d s .
\end{aligned}
$$

Similarly, by Lemma 2.2 and the inequality (23), we obtain

$$
\begin{aligned}
(E r r)_{4} & =\epsilon_{0} \omega_{p}^{2}\left(\boldsymbol{E}^{I}-\boldsymbol{E}, \xi\right) \leq \epsilon_{0} \omega_{p}^{2} C h^{k+1}\|\boldsymbol{E}\|_{k+1}\|\xi\|_{0} \\
& \leq \delta_{4} \epsilon_{0} \omega_{p}^{2}\|\xi\|_{0}^{2}+\frac{\epsilon_{0} \omega_{p}^{2} C h^{2(k+1)}}{4 \delta_{4}}\|\boldsymbol{E}\|_{k+1}^{2}
\end{aligned}
$$

and

$$
\begin{aligned}
(E r r)_{5} & =-\epsilon_{0} \omega_{p}^{2} \int_{0}^{t}\left(\left(\boldsymbol{E}^{I}-\boldsymbol{E}\right)_{t}, \xi\right) d s \leq \epsilon_{0} \omega_{p}^{2} \int_{0}^{t} C h^{k+1}\left\|\boldsymbol{E}_{t}\right\|_{k+1}\|\xi\|_{0} d s \\
& \leq \int_{0}^{t} \delta_{5} \epsilon_{0} \omega_{p}^{2}\|\xi\|_{0}^{2} d s+\frac{\epsilon_{0} \omega_{p}^{2} C h^{2(k+1)}}{4 \delta_{5}} \int_{0}^{t}\left\|\boldsymbol{E}_{t}\right\|_{k+1}^{2} d s .
\end{aligned}
$$


Note that

$$
\begin{aligned}
\left(\boldsymbol{J}\left(\boldsymbol{E}-\boldsymbol{E}^{I}\right), \xi_{t}\right) & =\epsilon_{0} \omega_{p}^{2}\left(\int_{0}^{t} e^{-\nu(t-s)}\left(\boldsymbol{E}-\boldsymbol{E}^{I}\right)(\mathbf{x}, s) d s, \xi_{t}(\mathbf{x}, t)\right) \\
& \leq \epsilon_{0} \omega_{p}^{2} \int_{0}^{t} C h^{k+1}\|\boldsymbol{E}\|_{k+1}\left\|\xi_{t}\right\|_{0} d s
\end{aligned}
$$

from which we have

$$
\begin{aligned}
(E r r)_{6} & =\nu \int_{0}^{t}\left(\boldsymbol{J}\left(\boldsymbol{E}-\boldsymbol{E}^{I}\right), \xi_{t}\right) d s \leq \nu \epsilon_{0} \omega_{p}^{2} t \int_{0}^{t} C h^{k+1}\|\boldsymbol{E}\|_{k+1}\left\|\xi_{t}\right\|_{0} d s \\
& \leq \int_{0}^{t} \delta_{6} \epsilon_{0}\left\|\xi_{t}\right\|_{0}^{2} d s+\frac{\epsilon_{0} \omega_{p}^{4} t^{2} C h^{2(k+1)}}{4 \delta_{6}} \int_{0}^{t}\|\boldsymbol{E}\|_{k+1}^{2} d s .
\end{aligned}
$$

Finally, using the inequality (23), we have

$$
\begin{aligned}
(E r r)_{7} & =\nu \int_{0}^{t}\left(\boldsymbol{J}(\xi), \xi_{t}\right) d s \leq \int_{0}^{t} \delta_{7} \epsilon_{0}\left\|\xi_{t}\right\|_{0}^{2} d s+\frac{\nu^{2}}{4 \delta_{7} \epsilon_{0}} \int_{0}^{t}\|\boldsymbol{J}(\xi)\|_{0}^{2} d s \\
& \leq \int_{0}^{t} \delta_{7} \epsilon_{0}\left\|\xi_{t}\right\|_{0}^{2} d s+\frac{\nu \epsilon_{0} \omega_{p}^{4} t}{8 \delta_{7}} \int_{0}^{t}\|\xi\|_{0}^{2} d s
\end{aligned}
$$

where in the last step we used the estimate (25).

Substituting the above estimates for $(E r r)_{i}$ into (63) , choosing constants $\delta_{2}, \delta_{4}<$ $\frac{1}{2}$ and absorbing the first terms in $(E r r)_{2}$ and $(E r r)_{4}$ by the corresponding terms on the left hand side of (63), we obtain

$$
\begin{aligned}
\frac{1}{2}\left(\epsilon_{0}\left\|\xi_{t}\right\|_{0}^{2}+\mu_{0}^{-1}\|\nabla \times \xi\|_{0}^{2}+\epsilon_{0} \omega_{p}^{2}\|\xi\|_{0}^{2}\right) \\
\leq C_{1} \int_{0}^{t}\left(\epsilon_{0}\left\|\xi_{t}\right\|_{0}^{2}+\mu_{0}^{-1}\|\nabla \times \xi\|_{0}^{2}+\epsilon_{0} \omega_{p}^{2}\|\xi\|_{0}^{2}\right) d s \\
\quad+C_{2} h^{2(k+1)} \int_{0}^{t}\left(\left\|\boldsymbol{E}_{t t}\right\|_{k+1}^{2}+\left\|\boldsymbol{E}_{t}\right\|_{k+2}^{2}+\|\boldsymbol{E}\|_{k+1}^{2}\right) d s \\
\quad+C_{3} h^{2(k+1)}\|\boldsymbol{E}\|_{k+2}^{2},
\end{aligned}
$$

where we absorbed the explicit dependence of physical parameters into the generic constants $C_{1}, C_{2}$ and $C_{3}$.

Our proof concludes by using the Gronwall inequality (Lemma 2.3) to (64).

Using the postprocessing operator $\hat{\Pi}_{2 h}$ defined in the last section, we can easily achieve the following global superconvergence for the standard finite element method:

\section{Theorem 5.2.}

$$
\begin{aligned}
& \left\|\hat{\Pi}_{2 h} \boldsymbol{E}^{h}-\boldsymbol{E}\right\|_{0}+\left\|\left(\hat{\Pi}_{2 h} \boldsymbol{E}^{h}-\boldsymbol{E}\right)_{t}\right\|_{0} \\
& \quad \leq C h^{k+1}\left[\|\boldsymbol{E}\|_{k+2}+\left\|\boldsymbol{E}_{t}\right\|_{k+1}+\left(\int_{0}^{t}\left(\left\|\boldsymbol{E}_{t t}\right\|_{k+1}^{2}+\left\|\boldsymbol{E}_{t}\right\|_{k+2}^{2}+\|\boldsymbol{E}\|_{k+1}^{2}\right) d s\right)^{1 / 2}\right],
\end{aligned}
$$

where $k$ is the degree of edge elements in the space $\boldsymbol{V}_{h}$.

Remark 5.1. When $\Omega$ is not a cubic domain, global superconvergence of order $O\left(h^{k+\frac{1}{2}}\right)$ can be achieved on the almost cubic meshes by easily extending the results of Lin and Yan [29, p. 175]. 


\section{ACKNOWLEDGEMENTS}

Jichun Li thanks Dr. Miguel Visbal at the U.S. Air Force Research Laboratory (AFRL) for inspiring him to study the interesting dispersive media problems during his summer research at AFRL. The authors wish to thank the referees for many constructive comments that improved the paper.

\section{REFERENCES}

[1] M. Ainsworth, P. Davies, D. Duncan, P. Martin and B. Rynne, Topics in computational wave propagation, Lecture Notes in Computational Science and Engineering 31, Springer-Verlag, Berlin, 2003. MR2032865 (2004h:65008)

[2] A. Bossavit, Computational Electromagnetism, Academic Press, San Diego, 1998. MR 1488417 (99m:78001)

[3] C. Carstensen, S. Funken, W. Hackbusch, Ronald H.W. Hoppe and P. Monk (eds.), Computational Electromagnetics, Lecture Notes in Computational Science and Engineering 28, Springer-Verlag, Berlin, 2003. MR,1995092 (2004b:78001)

[4] C.M. Chen and Y.Q. Huang, High Accuracy Theory of Finite Element Methods (in Chinese), Hunan Science Press, China, 1995.

[5] M.-H. Chen, B. Cockburn and F. Reitich, High-order RKDG methods for computational electromagnetics, J. Sci. Comp. 22/23 (2005) 205-226. MR2142195 (2005m:65208)

[6] Q. Chen, M. Katsurai and P.H. Aoyagi, An FDTD formulation for dispersive media using a current density, IEEE Trans. Antennas Propagat. 46 (1998) 1739-1746.

[7] Z. Chen, Q. Du and J. Zou, Finite element methods with matching and nonmatching meshes for Maxwell equations with discontinuous coefficients, SIAM J. Numer. Anal. 37 (2000) 15421570. MR1759906 (2001h:78044)

[8] P. Ciarlet, Jr. and J. Zou, Fully discrete finite element approaches for time-dependent Maxwell's equations, Numer. Math. 82 (1999) 193-219. MR.1685459 (2000c:65083)

[9] B. Cockburn, F. Li and C.-W. Shu, Locally divergence-free discontinuous Galerkin methods for the Maxwell equations, J. Comp. Phys. 194 (2004) 588-610. MR2034859 (2004j:78024)

[10] G.C. Cohen, E. Heikkola, P. Joly and P. Neittaanmäki (eds.), Mathematical and numerical aspects of wave propagation - WAVES 2003, Springer-Verlag, Berlin, 2003. MR2077970 (2005b:00020)

[11] S.A. Cummer, An analysis of new and existing FDTD methods for isotropic cold plasma and a method for improving their accuracy, IEEE Trans. Antennas Propagat. 45 (1997) 392-400.

[12] L. Demkowicz, Fully automatic hp-adaptivity for Maxwell's equations, Comput. Methods Appl. Mech. Engrg. 194 (2005) 605-624. MR2105184 (2005m:65296)

[13] L. Demkowicz, P. Monk, Ch. Schwab and L. Vardapetyan, Maxwell eigenvalues and discrete compactness in two dimensions, Comput. Math. Appl. 40 (2000) 589-605. MR 1772657 (2002e:65166)

[14] R.E. Ewing, Y. Lin, T. Sun, J. Wang, and S. Zhang, Sharp $L^{2}$-error estimates and superconvergence of mixed finite element methods for non-Fickian flows in porous media, SIAM J. Numer. Anal. 40 (2002) 1538-1560. MR1951906 (2003m:65177)

[15] R. Hiptmair, Finite elements in computational electromagnetism, Acta Numerica 11 (2002) 237-339. MR2009375 (2004k:78028)

[16] P. Houston, I. Perugia and D. Schötzau, Energy norm a posteriori error estimation for mixed discontinuous Galerkin approximations of the Maxwell operator, Comput. Methods Appl. Mech. Engrg. 194 (2005) 499-510. MR2105178 (2005h:78027)

[17] D. Jiao and J.-M. Jin, Time-domain finite-element modeling of dispersive media, IEEE Microwave and Wireless Components Letters 11 (2001) 220-222.

[18] J. Jin, The Finite Element Method in Electromagnetics, 2nd ed., John Wiley \& Sons, Inc., New York, 2002. MR1903357(2004b:78019)

[19] M. Krizek and P. Neittaanmaki, Bibliography on Superconvergence, in "Finite Element Methods", Lecture Notes in Pure and Applied Math., Vol. 196, 1998, pp. 315-348. MR 1602730

[20] J. Li, Error analysis of finite element methods for 3-D Maxwell's equations in dispersive media, Journal of Computational and Applied Mathematics, 188 (2006) 107-120. MR 2192630 (2006m:65279) 
[21] J. Li and Y. Chen, Analysis of a time-domain finite element method for 3-D Maxwell's equations in dispersive media, Comput. Methods Appl. Mech. Engrg. 195 (2006) 4220-4229. MR 2229840

[22] J. Li, Error analysis of fully discrete mixed finite element schemes for 3-D Maxwell's equations in dispersive media, preprint, Sept. 2005.

[23] J. Li and M.F. Wheeler, Uniform convergence and superconvergence of mixed finite element methods on anisotropically refined grids, SIAM J. Numer. Anal. 38 (2000) 770-798. MR:1781203 (2001f:65137)

[24] Q. Lin, J. Li and A. Zhou, rectangle test for Ciarlet-Raviart scheme, Prof. of Sys. Sci. \& Sys. Engrg., Great Wall (H.K.) Culture Publish Co., 1991, pp. 230-231.

[25] Q. Lin, J. Li and A. Zhou, A rectangle test for the Stokes equations, Prof. of Sys. Sci. \& Sys. Engrg., Great Wall (H.K.) Culture Publish Co., 1991, pp. 240-241.

[26] Q. Lin and J.F. Lin, High accuracy approximation of mixed finite element for 2-D Maxwell equations. (Chinese) Acta Math. Sci. Ser. A Chin. Ed. 23 (2003), no. 4, 499-503. MR2090553 (2005i:65145)

[27] Q. Lin and J. Xu, Linear finite elements with high accuracy, J. Comp. Math. 3 (1985) 115-133. MR854355 (87k:65141)

[28] Q. Lin and N. Yan, Superconvergence of mixed element methods for Maxwell's equations (in Chinese), Gongcheng Shuxue Xuebao 13 (1996), suppl., 1-10. MR.1437477 (98c:65186)

[29] Q. Lin and N. Yan, Global superconvergence for Maxwell's equations, Math. Comp. 69 (1999) 159-176. MR1654029 (2000i:65131)

[30] Q. Lin and N. Yan, The Construction and Analysis of High Accurate Finite Element Methods (in Chinese), Hebei University Press, Hebei, China, 1996.

[31] Q. Lin, N. Yan and A. Zhou, A rectangle test for interpolated finite elements, Prof. of Sys. Sci. \& Sys. Engrg., Great Wall (H.K.) Culture Publish Co., 1991, pp. 217-229.

[32] T. Lu, P. Zhang and W. Cai, Discontinuous Galerkin methods for dispersive and lossy Maxwell's equations and PML boundary conditions, J. Comp. Phys. 200 (2004) 549-580. MR 2095277 (2005e:78025)

[33] P. Monk, An analysis of Nédélec's method for the special discretization of Maxwell's equations, J. Comp. Appl. Math. 47 (1993) 101-121. MR1226366 (94g:65105)

[34] P. Monk, Superconvergence of finite element approximations to Maxwell's equations, Numer. Methods Partial Differential Equations 10 (1994) 793-812. MR.1298123 (95h:65090)

[35] P. Monk, Finite element methods for Maxwell's equations, Oxford University Press, 2003. MR.2059447 (2005d:65003)

[36] J.-C. Nédélec, Mixed finite elements in $R^{3}$, Numer. Math. 35 (1980) 315-341. MR.592160 (81k:65125)

[37] A. Quarteroni and A. Valli, Numerical Approximation of Partial Differential Equations, Springer-Verlag, Berlin, 1994. MR.1299729 (95i:65005)

[38] A. Taflove and C. Hagness, Computational Electrodynamics: The Finite-Difference TimeDomain Method, 2nd ed., Artech House, Norwood, MA, 2000. MR1338377 (96f:78001)

[39] L.B. Wahlbin, Superconvergence in Galerkin Finite Element Methods, Springer, Berlin, 1995. MR 1439050 (98j:65083)

[40] Z. Zhang, N. Yan and T. Sun, Superconvergent derivative recovery for the intermediate finite element family of the second order, IMA Journal of Numerical Analysis 21 (2001) 643-665. MR:1844362(2002f:65171)

LSEC, ICMSEC, Academy of Mathematics and Systems Science, Chinese Academy of Sciences, Beijing 100080, China

E-mail address: linq@lsec.cc.ac.cn

Department of Mathematical Sciences, University of Nevada, Las Vegas, 4505 Maryland Parkway, Box 454020, Las Vegas, Nevada 89154-4020

E-mail address: jichun@unlv.nevada.edu 